\title{
El Principio de Activación en el Pensamiento Computacional, las Matemáticas y el STEM ${ }^{1}$
}

\section{The Activation Principle in Computational Thinking, Mathematics, and STEM $^{2}$}

\author{
José Miguel Zapata, \\ University College of Dublin. Dublin. Irlanda \\ jose.zapatagarcia@ucd.ie \\ Ellen Jameson, \\ University of Cambridge. Cambridge. UK \\ Ellen.Jameson@Cambridgemaths.org \\ Miguel Zapata-Ros, \\ Universidad de Murcia. Murcia. España. \\ mzapata@um.es \\ David Merrill, \\ Utah State University. Logan. EE UU \\ professordavemerrill@gmail.com
}

\begin{abstract}
Resumen. -
El presente número monográfico de RED se convocó con el título La educación en Matemáticas, Pensamiento Computacional y STEM apoyada por la tecnología digital. Su diseño instruccional. El principio de activación. En este artículo los autores queremos hacer la presentación del número haciendo énfasis en el principio de activación como fundamento teórico del pensamiento computacional y de la educación matemática y de STEM
\end{abstract}

Hay numerosos destrezas y conceptos, propios de Pensamiento Computacional, Matemáticas y STEM, que son necesarios como base para los estudios de grado. Frecuentemente estos conceptos y procedimientos no constan de forma explícita en el currículo de los niveles anteriores a la universidad o, constando, no se garantizado su dominio. En estos casos no podemos esperar que aparezcan de forma espontánea, en el mismo momento en que se necesitan para los contenidos de grado o de secundaria superior. Es necesaria una sólida base cognitiva para que puedan ser evocados.

Así pues, este número está convocado con el objetivo de dar oportunidad de difundir investigaciones y casos que parciales o totalmente tengan como referencia esas ideas. E investiguen si efectivamente el uso del principio de activación (Merrill, 2002; edición revisada 2020) convenientemente utilizado en un diseño instruccional adecuado, con métodos, actividades y recursos, consigue una mejora en la calidad de los resultados de aprendizaje cuando lo aprendido en las etapas anteriores es evocado y reactivado convenientemente en los estudios de grado.

La conclusión de todo ello es que debe potenciarse una pedagogía que establezca valores en estas ideas y principios para las primeras etapas educativas.

El principio de activación es pues clave para tenerlo en cuenta cuando se diseña la educación infantil y el primer ciclo de primaria, teniendo en el futuro los aprendizajes de horizonte, incluidos los de STEM.

\footnotetext{
${ }^{1}$ Presentación del número especial

${ }^{2}$ Presentation of the special issue
} 
Palabras clave. -

Principio de activación, principales principios del aprendizaje, pensamiento computacional, educación matemática, STEM

\begin{abstract}
. -
This monographic issue of RED was convened with the title Education in Mathematics, Computational Thinking and STEM supported by digital technology. Its instructional design. The activation principle.

There are numerous skills and concepts, specific to Computational Thinking, Mathematics and STEM, that are necessary as a basis for undergraduate studies.

Often times these concepts and procedures are not explicitly stated in the primary and secondary school curriculum. Other times it does appear but its mastery is not guaranteed. We cannot expect these skills to appear spontaneously, at the very moment they are needed. A solid cognitive foundation is necessary for them to be activated.
\end{abstract}

This is the presentation of the special issue in order to present research and experiences on Computational Thinking and the Principle of Activation in STEM

\title{
Key words. -
}

Activation principle, first principles of learning, computational thinking, mathematics education, STEM

El presente número especial de RED se convocó con el título La educación en Matemáticas, Pensamiento Computacional y STEM apoyada por la tecnología digital. Su diseño instruccional. El principio de activación.

Hay numerosas destrezas y conceptos, propios de Pensamiento Computacional, Matemáticas y STEM, que son necesarios como base para los estudios de grado. Frecuentemente estos conceptos y procedimientos no constan de forma explícita en el currículo de los niveles anteriores a la universidad o, constando, no se garantizado su dominio. En estos casos no podemos esperar que aparezcan de forma espontánea, en el mismo momento en que se necesitan para los contenidos de grado o de secundaria superior. Es necesaria una sólida base cognitiva para que puedan ser activados.

Así pues, este número monográfico está convocado con el objetivo de dar oportunidad a investigaciones y casos, que parcial o totalmente tengan como referencia esas ideas, de ser difundidas. E investiguen si efectivamente el uso del principio de activación (Merrill, 2002; 2020 revised edition) convenientemente utilizado en un diseño instruccional adecuado, con métodos, actividades y recursos, consigue una mejora en la calidad en los resultados de aprendizaje, cuando lo aprendido en las etapas anteriores es evocado y reactivado convenientemente en los estudios de grado.

El Principio de Activación en el Pensamiento Computacional, las Matemáticas y el STEM. José Miguel Zapata, Ellen Jameson, Miguel Zapata-Ros y David Merrill. 
Finalmente, sólo se han recibido dos trabajos con ese fin. Ello no exime a la revista de justificar la importancia de ese planteamiento y a dar la bienvenida a esos primeros trabajos, de los cuales no dudamos habrá continuación en el futuro.

Las habilidades que son necesarias para la programación de algoritmos complejos, las destrezas del pensamiento computacional en todo su vigor (análisis descendente, análisis ascendente, métodos de aproximaciones sucesivas, ensayo-error, ....) y las que entraña la operatividad lógica o abstracta que son necesarias para la programación de ordenadores, para resolver problemas, no podemos esperar a que aparezcan, o a que se manifiesten de forma espontánea. Y que lo hagan en el mismo momento de necesitarlas en los estudios de grado de Matemáticas, Computación o Ingeniería, en la etapa de madurez del alumno que corresponde a esa edad, ni tan siquiera en la etapa de desarrollo del pensamiento abstracto, en la secundaria postobligatoria o incluso en Secundaria.

En esto, estas habilidades no son distintas de otras habilidades complejas que tienen que ver con el desarrollo de los individuos, que se adquieren de forma progresiva y que solo son utilizables en forma operativa en su última fase.

Esta naturaleza del aprendizaje, el enlace de las situaciones en que se produce, o se debiera producir, con los objetivos finales a través de etapas, niveles y condiciones de aprendizaje, es la que justifica el diseño instruccional y de ello no se libra la adquisición de las habilidades de STEM ni, siendo distinto, el pensamiento computacional. Los aprendizajes complejos se dividen, se fraccionan en aprendizajes más simples, más cercanos a las capacidades de los individuos y más lejanos del momento que adquieren su mayor eficiencia u operatividad práctica. Esto es válido incluso en el caso en que no alcancen esa eficacia por sí mismos, sino como habilidades auxiliares a otras. Así pasa con los conocimientos y las habilidades básicas y con las competencias clave, entre las que tradicionalmente se encuentras la escritura, la lectura y la aritmética (las "tres erres")

Es este el punto donde obtienen su justificación las teorías del aprendizaje, particularmente el principio de activación de Merrill (2002; 2020 revised edition), en la forma en como transitar desde que se adquieren las habilidades hasta que son útiles en su destino final. Este tránsito y la forma de organizarlo es lo que constituye la base del diseño instruccional. Por tanto, son dos núcleos clave que está en la justificación en la teoría del aprendizaje y en la base de una pedagogía del STEM: El principio de activación y el diseño instruccional.

En este caso nos vamos a dedicar exclusivamente al principio de activación.

Así pues, vamos a justificar con este principio la necesidad y la conveniencia de trabajar aspectos del aprendizaje previos y necesarios para todos los contenidos de STEM en la Educación Superior que conviene adquirir desde las primeras etapas del desarrollo cognitivo de los individuos.

Estas ideas y resultados son los que van a justificar después qué actividades constituyen el currículo (así, por ejemplo, entre ellas incluiremos juegos, tarjetas, escarabajos robots, etc.) y cómo se organizan. También nos informan sobre cómo lo hacemos para que los conceptos y preconceptos, en el sentido que Piaget (1973) y Piaget y Herborth (1973), a través de Burgmann y Wille (2006), y Vosniadou (2012) los definen,

El Principio de Activación en el Pensamiento Computacional, las Matemáticas y el STEM. José Miguel Zapata, Ellen Jameson, Miguel Zapata-Ros y David Merrill. 
se activen y fluyan en la fase de resolver problemas en las etapas de enseñanza universitaria.

Esto es en lo que se refiere a lo que hay que trabajar en las primeras etapas para que pueda ser después activado. Pero hay otros conceptos que no son en principio trabajados en esas etapas básicas, y sí merecen la pena ser tenidos en cuenta en las etapas superiores, cuando se produce esa activación. Son los conceptos ingenuos. Así pues, en esa fase hemos de estudiar y tener en cuenta en el diseño instruccional: cómo lo hacemos para que se activen los conceptos ingenuos de los estudiantes (Özdemir \& Clarke, 2007), además de los preconceptos (Vosniadou, 2012).

Esto obviamente sería una ejemplificación extrema. En un caso más normal, la adquisición se produciría de una forma más progresiva. A través de las distintas etapas educativas, de los niveles. E incluso en el trascurrir de éstos y de los módulos y unidades instruccionales que los componen.

En su trabajo, Merrill (2002 y 2020 revised edition) desarrolla lo que llama unos principios fundamentales del aprendizaje (first priciples) lo hace decantando los principios subyacentes en los que hay consensos, hay un acuerdo esencial, en todas las teorías y que previamente ha identificado.

Ese trabajo está expuesto y desarrollado en su artículo First principles of instruction (Merrill, 2002), en Educational technology research and development, incluido también como capítulo en el tercer volumen de los libros de Reigueluth Instructional-design theories and models: Building a common knowledge base (Merrill, 2009). Y de forma resumida en First principles of instruction: A synthesis (Merrill, 2007).

Todo ello está revisado y actualizado en el último libro de Merrill (2020): First principles of instruction (revised edition).

También son glosados como base del nuevo paradigma instruccional de Reigeluth, cuya versión oficial pueden encontrar en RED número 50, en el artículo Teoría instruccional y tecnología para el nuevo paradigma de la educación (Reigeluth, 2016).

En este último trabajo, Reigeluth (2016) distingue entre principios universales y escenarios particulares. Cuando aplicamos con mayor precisión un principio o un método instruccional, por lo general descubrimos que hace falta que éste sea diferente para diferentes situaciones y perfiles de aprendizaje o, en una mayor precisión, para obtener objetivos contextualizados y personalizados. Reigeluth (1999) se refirió a los factores contextuales que influyen en los efectos de los métodos como "escenarios".

Los principios fundamentales de instrucción (first priciples) los propone y los define Merrill (2002) en First principles of instruction. Este documento se refiere a los métodos variables como programas y prácticas. Un principio fundamental (Merrill, 2002), o un método básico según Reigueluth (1999a), es un aserto que siempre es verdadero bajo las condiciones apropiadas independientemente del programa o de la práctica en que se aplique, que de esta forma dan lugar a un método variable. Teniendo en cuenta como el mismo Merrill (2002) las define:

El Principio de Activación en el Pensamiento Computacional, las Matemáticas y el STEM. José Miguel Zapata, Ellen Jameson, Miguel Zapata-Ros y David Merrill. 
Una práctica es una actividad instruccional específica. Un programa es un enfoque que consiste en un conjunto de prácticas prescritas. Las prácticas siempre implementan o no implementan los principios subyacentes ya sea que estos principios se especifiquen o no. Un enfoque de instrucción dado solo puede enfatizar la implementación de uno o más de estos principios de instrucción. Los mismos principios pueden ser implementados por una amplia variedad de programas y prácticas.

De esta forma Merrill propuso un conjunto de cinco principios instruccionales prescriptivos (o "principios fundamentales") que mejoran la calidad de la enseñanza en todas las situaciones Esos principios tienen que ver con la centralidad de la tarea, la activación, la demostración, la aplicación y la integración.

Para ello Merrill (2002) propone un esquema en fases como el más eficiente para el aprendizaje, de manera que centran el problema y crean un entorno que implica al alumno para la resolución de cualquier problema En cuatro fases distintas, cuando habitualmente solo se hace en una: la de demostración, reduciendo todo el problema a que el alumno pueda demostrar su conocimiento o su habilidad en la resolución del problema en una última fase. Son las FASES DE INSTRUCCIÓN (ver Figura 1).

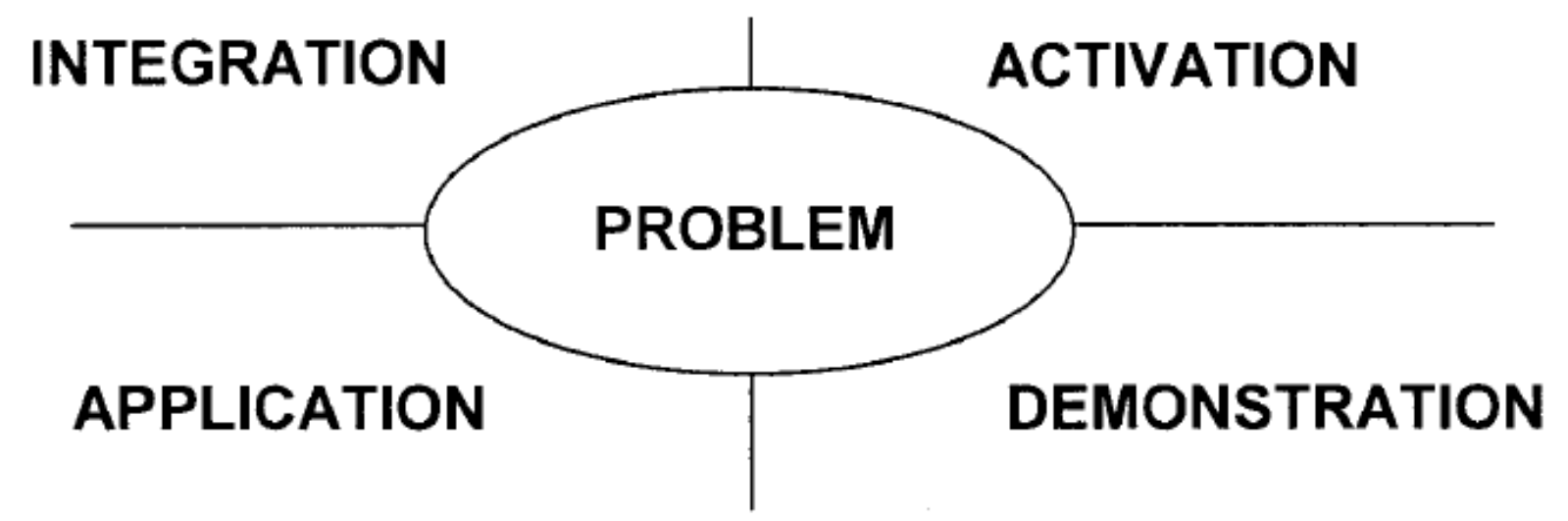

Figura 1. Fases de la instrucción según Merrill (2002)

Las fases son (a) activación de experiencia previa, (b) demostración de habilidades, (c) aplicación de habilidades, y (d) integración de estas habilidades en actividades del mundo real.

Así la figura anterior proporciona un marco conceptual para establecer y relacionar los principios fundamentales de la instrucción. De ellos uno tiene que ver con la implicación y la naturaleza real del problema, así percibida por el alumno, y los cuatro restantes para cada una de las fases. Así estos cinco principios enunciados en su forma más concisa (Merrill 2002) son:

1. El aprendizaje se promueve cuando los estudiantes se comprometen a resolver

El Principio de Activación en el Pensamiento Computacional, las Matemáticas y el STEM. José Miguel Zapata, Ellen Jameson, Miguel Zapata-Ros y David Merrill.

Página 5 de 9 
problemas del mundo real. Es decir, el aprendizaje se promueve cuando es un aprendizaje centrado en la tarea.

2. El aprendizaje se favorece cuando existen conocimientos que se activan como base para el nuevo conocimiento.

3. El aprendizaje se promueve cuando se centra en que el alumno debe demostrar su nuevo conocimiento. Y el alumno es consciente de ello.

4. El aprendizaje se promueve igualmente cuando se centra en que el aprendiz aplique el nuevo conocimiento.

5. El aprendizaje se favorece cuando el nuevo conocimiento se tiende a que se integre en el mundo del alumno.

Pero, de todos estos principios, el que justifica sobremanera la inclusión del pensamiento computacional, como pensamiento computacional desenchufado en las primeras etapas, es el principio de activación. En él nos vamos a centrar, y no solo en su aplicación para el diseño instruccional en la fase de activación, en la que el conocimiento existente se activa, sino en las fases en las que se crean los conocimientos y habilidades que son activados, y en cómo hacerlo para que la activación sea más eficiente.

En su trabajo Teoría instruccional y tecnología para el nuevo paradigma de la educación, Reigeluth (2016 pág. 4) caracteriza el principio de activación de manera que:

- El diseño educativo de actividades, organización, recursos, etc. debe ser tendente a activar en los alumnos estructuras cognitivas relevantes, haciéndoles recordar, describir o demostrar conocimientos o experiencias previas que sean relevantes para él.

- La activación puede ser social. La instrucción debe lograr que los estudiantes compartan sus experiencias anteriores entre ellos.

- La instrucción debe hacer que los estudiantes recuerden o adquieran una estructura para organizar los nuevos conocimientos.

Los trabajos de Merrill (2002 y 2020) y Reigeluth (2016) hacen énfasis en la fase de activación, pero no en la fase de crear estructuras cognitivas, experiencias y en general conocimientos y habilidades que puedan ser evocados. Ni tampoco en crear una pedagogía o un diseño educativo que incluya, o tendente a favorecer, elementos cognitivos de enlace que promuevan la activación. Tampoco a fomentar la investigación sobre estos temas, o a investigar qué tipos de enlaces fortalecen más las estructuras cognitivas de enlace y de activación.

A partir de lo que dicen, sobre las características del diseño instruccional que implica el principio de activación, los ítems anteriores, podemos concluir que la instrucción, en la fase de crear elementos para ser evocadora, debe:

El Principio de Activación en el Pensamiento Computacional, las Matemáticas y el STEM. José Miguel Zapata, Ellen Jameson, Miguel Zapata-Ros y David Merrill. 
- Crear estructuras cognitivas que incluyan conocimientos, habilidades, elementos de reconocimiento que permitan distinguir al alumno y otorgar relevancia en su momento de forma fluida a esas habilidades para conseguir su efectividad en ese momento a partir de elementos contextuales, metáforas, etc.

- Otorgar a esas habilidades elementos de reconocimiento que permitan la activación.

- Asociar esas habilidades a tareas que tengan similitud con las que se en su momento sean necesarias para resolver los problemas a los que ayuda la evocación. En nuestro caso, a los problemas computacionales, o habilidades propias a los elementos que constituyen el pensamiento computacional.

- Diseñar instruccionalmente las actividades que sean relevantes para evocar los elementos de pensamiento computacional (Pérez-Paredes y Zapata-Ros, 2018).

- Propiciar experiencias de aprendizaje compartido en las primeras etapas y hacer que esos grupos y experiencias sociales sean estables a lo largo del tiempo. Las experiencias compartidas crean elementos de activación a través de grupos o de pares alumnos. El propiciar grupos y claves de comunicación, de lenguaje, y que esos grupos sean estables a lo largo del tiempo aumenta la potencia de activación.

- Crear estructuras cognitivas en los alumnos capaces de recomponerse y aumentar en el futuro. Dotar a los conocimientos y habilidades de referencias y de metadatos que permitan ser recuperados mediante activación.

Debe pues potenciarse una pedagogía que establezca valores en estas ideas y principios para las primeras etapas.

El principio de activación es pues clave para tenerlo en cuenta cuando se diseña la educación infantil y del primer ciclo de primaria teniendo en el horizonte los aprendizajes futuros, incluidos los de STEM.

Además de Merrill ha habido más aportaciones sobre el principio de activación y el aprendizaje STEM.

Como señalamos en otro trabajo (Zapata-Ros, 2018b), Bawden (2008) refiriéndonos a Pensamiento Computacional, se habla de habilidades de recuperación, y se remite a lo expuestas en otro trabajo anterior (Bawden, 2001). En las habilidades que señala se constatan ideas como la de construir un "bagaje de información fiable" de diversas fuentes, la importancia de las habilidades de recuperación, utilizando una forma de "pensamiento metacognitivo" para hacer juicios informados sobre la información recuperada, y para asegurar la validez e integridad de las fuentes de Internet, leer y comprender de forma dinámica y cambiante material no secuencial.

Para concluir, no podemos finalizar esta presentación sin agradecer a Chales M. Reigeluth su participación y aliento en todas las fases del monográfico. Primero con su producción teórica poniendo en relación los principios principales del aprendizaje con el diseño instruccional en todas las fases niveles y programas educativos, y particularmente

El Principio de Activación en el Pensamiento Computacional, las Matemáticas y el STEM. José Miguel Zapata, Ellen Jameson, Miguel Zapata-Ros y David Merrill. 
en Pensamiento Computacional. Y luego con su implicación en la elaboración del número, invitando a quien hoy es autor de esta presentación, Dave Merrill, a participar. También propiciando la participación de autores con su implicación personal en la difusión y valoración de las ideas que se quieren potenciar sobre el principio de activación y el pensamiento computacional.

También agradecemos al Profesor Richard West, de la BYU, su apoyo difundiendo la iniciativa y animando a investigadores no sólo en participar en este número, lo cual era difícil si previamente esta idea no contaba, como ha sucedido, con la suficiente tradición y producción, sino sobre todo a sembrar inquietudes para propiciar futuras investigaciones.

Presentación del artículo: 1 de julio 2021

Fecha de aprobación: 9 de noviembre de 2021

Fecha de publicación: 30 de noviembre de 2021

Zapata, J.M., Jameson, E., Zapata-Ros, M. y Merrill, M. (2021). El Principio de Activación en el Pensamiento Computacional, las Matemáticas y el STEM. RED. Revista de Educación a Distancia. Núm. 68, DOI: http://dx.doi.org/10.6018/red.498531

\section{Financiación}

Este trabajo no ha recibido ninguna subvención específica de los organismos de financiación en los sectores públicos, comerciales o sin fines de lucro

\section{Referencias}

Bawden, D. (2001). Information and digital literacies: a review of concepts. Journal of Documentation, 57(2), 218-259.

Bawden, D. (2008). Origins and concepts of digital literacy. Digital literacies:

Concepts, policies and practices, 17-32.

http://sites.google.com/site/colinlankshear/DigitalLiteracies.pdf\#page=19

Burgmann, C., \& Wille, R. (2006). The basic theorem on preconcept lattices. In Formal Concept Analysis (pp. 80-88). Springer, Berlin, Heidelberg.

https://link.springer.com/chapter/10.1007/11671404_5

https://link.springer.com/content/pdf/10.1007\%2F11671404.pdf

Merrill, M. D. (2002). First principles of instruction. Educational technology research and development, 50(3), 43-59.

https://link.springer.com/article/10.1007/BF02505024 y

https://mdavidmerrill.com/Papers/firstprinciplesbymerrill.pdf

Merrill, M. D. (2007). First principles of instruction: A synthesis. In R. A. Reiser \& J. V. Dempsey (Eds.), Trends and issues in instructional design and technology (2nd ed., pp. 62-71). Upper Saddle River, NJ: Merrill/Prentice-Hall.

El Principio de Activación en el Pensamiento Computacional, las Matemáticas y el STEM. José Miguel Zapata, Ellen Jameson, Miguel Zapata-Ros y David Merrill. 
Merrill, M. D. (2009). First principles of instruction. In C. M. Reigeluth \& A. A. CarrChellman (Eds.), Instructional-design theories and models: Building a common knowledge base (Vol. III, pp. 41-56). New York: Routledge.

Merrill, D. (2020). First principles of instruction (revised edition). Association for Educational Communications and Technology.

Özdemir, G., \& Clark, D. B. (2007). An overview of conceptual change theories. Eurasia Journal of Mathematics, Science and Technology Education, 3(4), 351-361.

Piaget J. (1973) Einf uhrung in die genetische Erkenntnistheorie. suhrkamp taschenbuch wissenschaft 6 . Suhrkamp.

Piaget, J., \& Herborth, F. (1973). Einführung in die genetische Erkenntnistheorie. Frankfurt am Main: Suhrkamp.

Reigeluth, C. M. (1999). What is instructional-design theory and how is it changing? In C. M. Reigeluth (Ed.), Instructional-design theories and models: A new paradigm of instructional theory (Vol. II, pp. 5-29). Mahwah, NJ: Lawrence Erlbaum Associates.

Reigeluth, C. M. (2016). Teoría instruccional y tecnología para el nuevo paradigma de la educación. RED. Revista de Educación a Distancia. Número 50. http://www.um.es/ead/red/50

Vosniadou, S. (2012). Reframing the classical approach to conceptual change: Preconceptions, misconceptions and synthetic models. In Second international handbook of science education (pp. 119-130). Springer, Dordrecht.

Zapata-Ros, M. (2018b). Pensamiento computacional. Una tercera competencia clave. (I) Blog RED El aprendizaje en la Sociedad del Conocimiento. https://red.hypotheses.org/1059 